\title{
Antimicrobial sonodynamic and photodynamic therapies against Candida albicans
}

\section{Fernanda Alves, Ana Cláudia Pavarina, Ewerton Garcia de Oliveira Mima,} Anthony P. McHale \& John Francis Callan

To cite this article: Fernanda Alves, Ana Cláudia Pavarina, Ewerton Garcia de Oliveira Mima, Anthony P. McHale \& John Francis Callan (2018) Antimicrobial sonodynamic and photodynamic therapies against Candida albicans, Biofouling, 34:4, 357-367, DOI: 10.1080/08927014.2018.1439935

To link to this article: https://doi.org/10.1080/08927014.2018.1439935

央 Published online: 19 Apr 2018.

Submit your article to this journal

Џ Article views: 252

View Crossmark data $₫$ 


\title{
Antimicrobial sonodynamic and photodynamic therapies against Candida albicans
}

\author{
Fernanda Alves ${ }^{\mathrm{a}}$, Ana Cláudia Pavarina ${ }^{\mathrm{a}}$ (D), Ewerton Garcia de Oliveira Mima ${ }^{\mathrm{a}}$, Anthony P. McHale ${ }^{\mathrm{b}}$ and John \\ Francis Callan ${ }^{\mathrm{b}}$ \\ aDepartment of Dental Materials and Prosthodontics, School of Dentistry, São Paulo State University (UNESP), Araraquara, Brazil; bSchool of \\ Pharmacy and Pharmaceutical Sciences, Faculty of Life \& Health Sciences, Ulster University, Coleraine, UK
}

ABSTRACT

Candida albicans biofilms exhibit unique characteristics and are highly resistant to antifungal agents. Antimicrobial photodynamic therapy (aPDT) is an alternative treatment limited to treating superficial infections due to the poor light penetration. In this manuscript, the antifungal properties of sonodynamic therapy (SDT) were assessed. SDT uses ultrasound instead of light, enabling the treatment of deeper infections. Planktonic cells and biofilms of $C$. albicans were treated with aPDT or SDT, in addition to combined aPDT/SDT, with cell survival determined using colony forming units. The total biomass and structural integrity of the biofilms were also investigated. The results demonstrated that while individual aPDT or SDT eradicated suspensions, they had little impact on biofilms. However, combined aPDT/SDT significantly reduced the viability and total biomass of biofilms. Microscopic images revealed that biofilms treated with aPDT/SDT were thinner and comprised mainly of dead cells. These results highlight the potential of combined aPDT/SDT for the inactivation of C. albicans biofilms.
ARTICLE HISTORY

Received 23 June 2017

Accepted 6 February 2018

\section{KEYWORDS}

Candida albicans;

photodynamic therapy;

sonodynamic therapy;

biofilms; antimicrobial; antifungal

\section{Introduction}

Oropharyngeal candidosis (OPC) is one of the most common manifestations of mucocutaneous candidiasis in patients receiving corticosteroid therapy, prolonged use of antibiotics, cancer chemotherapy and in those suffering from immunosuppression after transplantation or human immunodeficiency virus (HIV) infections (PeresBota et al. 2004). Depending on the stage of immunosuppression, up to $90 \%$ of HIV-positive patients present with OPC, which consequently is considered an indicator of the development of HIV infection (Pfaller and Diekema 2007). Candida albicans is the main etiological agent of OPC and the switching from commensal to the pathogenic condition has been widely known to be associated with its ability to grow in filamentous form that facilitates invasion of epithelial tissue (Sudbery 2011). Furthermore, Candida species have the ability to form a biofilm that is a highly structured community of microorganisms attached to a surface and are embedded in a self-produced protective extracellular matrix (Costerton et al. 1999; Ramage et al. 2009). The extracellular matrix contributes to the preservation of the biofilm architecture and to the maintenance of stable cell-cell, cell-surface and cell-environment interactions (Flemming and Wingender 2010). The benefits of organisms being organized as biofilms include protection from environmental changes, protection against host defense, enabling metabolic cooperation and regulation of gene expression (Ramage et al. 2009). For these reasons, cells in biofilms exhibit unique phenotypic characteristics compared to their planktonic counterparts and from a therapeutic perspective, tend to be more resistant to antimicrobial agents (Ramage et al. 2009). Candida spp. can also be associated with disseminated forms of disease, such as candidemia, which exhibit a high morbidity and mortality rate (Pfaller and Diekema 2007).

Considering the high frequency of Candida infections in immunocompromised patients, it is clear that an effective antifungal therapy is necessary. Topical antifungal agents are often prescribed to manage OPC (Lombardi and Budtz-Jorgensen 1993; Banting et al. 1995). However, these agents achieve only a transient response and relapses are frequent (Samaranayake and MacFarlane 1981; Kulak et al. 1994). As the recurrence rate is high, systemic azole antifungals (eg fluconazole and itraconazole) have mainly been used to treat fungal infections (Samaranayake and 
MacFarlane 1981; Barchiesi et al. 1998; Ellepola and Samaranayake 2000). Nevertheless, the increased use of azoles, combined with several cases of treatment failures, has drawn attention to the problem of antifungal resistance (Hunter et al. 1998; White et al. 1998; Goldman et al. 2004). Clinical resistance to antifungal drugs is a broad concept describing failure of an antifungal therapy, which results in persistence or progression of an infection (White et al. 1998). It has been reported that exposure to fluconazole led to the development of $C$. albicans resistance in HIV-positive patients (Hunter et al. 1998), and also led to the replacement of fluconazole-susceptible C. albicans strains with other species that are intrinsically less sensitive to fluconazole, such as Candida glabrata and Candida krusei (Hunter et al. 1998; White et al. 1998).

To overcome the problems associated with antifungal resistance, studies have been performed to search for new alternative therapies for resistant infections. One potential alternative approach is antimicrobial photodynamic therapy (aPDT). aPDT requires the association of oxygen, a visible light source and photosensitizer (PS) to exert antimicrobial activity (Bonnett and Martínez 2001). In general, the PS is applied externally to the cell, thus the cell membrane is considered the initial target of the photodynamic process (Strakhovskaia et al. 2002; Donnelly et al. 2008). Different PSs have been evaluated for the inactivation of Candida species through aPDT, including porphyrins (Bliss et al. 2004; Dovingo, Pavarina, Carmello et al. 2011; Mima et al. 2012), phenothiazine dyes (Paardekopper et al. 1992; Pereira et al. 2011), chlorins (Park et al. 2010; Dovigo et al. 2013), curcumin (Dovigo, Pavarina, Carmello et al. 2011) and rose bengal (RB) (Costa et al. 2012). However, studies have shown that Candida biofilms are less susceptible to aPDT when compared with their planktonic counterparts (Dovigo, Pavarina, Mima et al. 2011; Dovigo et al. 2013; Costa et al. 2012).

It has been demonstrated that certain PS can also be activated by ultrasound (US), and as a result, the PS may also be referred to as a sonosensitizer (SS). This approach is known as sonodynamic therapy (SDT) (Ma et al. 2009; Serpe and Giuntini 2015). The advantage of SDT when compared to aPDT is that US propagates deeper into the tissue than light; therefore, SDT may be used to treat deeper lesions overcoming the limitations of light propagation and delivery presented by aPDT (Ma et al. 2009; Costley et al. 2015; Serpe and Giuntini 2015). It has been suggested that in SDT, singlet oxygen generation may result by the indirect photo-activation of the sensitizer drug via sonoluminescence (Umemura et al. 1999). Once excited the sensitizer generates singlet oxygen in the same way as in the aPDT (Umemura et al. 1999; Hiraoka et al. 2006). An alternative hypothesis suggests that the elevated temperatures resulting from cavitation can result in the generation of radicals directly from the PS, and ROS are subsequently produced in much the same way as a Type I photodynamic reaction.

The use of SDT as an antimicrobial treatment to overcome antimicrobial resistance is a relatively new approach and publications in the field are rare. A number of studies in this area have reported US-mediated inactivation of bacteria using different sensitizers, including rose-bengal (RB) (Nakonechny et al. 2013; Costley et al. 2017), curcumin (Wang et al. 2014) and porphyrin (Zhuang et al. 2014).

In this manuscript, the ability of aPDT, SDT and a combination of these treatments was evaluated, using the sensitizers RB and Photodithazine, to inactivate C. albicans. Cell viability was assessed by colony forming units (CFU), the total biomass of the biofilm was measured by crystal violet staining and biofilm architecture was analyzed by light and fluorescence microscopy.

\section{Materials and methods}

\section{Sensitizers, light sources and ultrasound device}

The chlorin e6 derivative Photodithazine (PDZ, produced by Veta-Grand Co., Moskva, Russia) and RB (SigmaAldrich, St Louis, MO, USA) were used as sensitizers. The PDZ has I band absorption maxima at longer wavelengths (650-670 nm). Prior to the beginning of each experiment, the work solution of PDZ was obtained by diluting the commercial solution of PDZ (5,000 $\left.\mathrm{mg} \mathrm{l}^{-1}\right)$ in physiological saline $(0.85 \% \mathrm{NaCl})$. RB has an absorption maximum of $560 \mathrm{~nm}$ and was also diluted in physiological solution. A range of concentrations of each sensitizer was evaluated.

The PDZ sensitizer was irradiated by a light emitting diode (LED) in the red region of the spectrum (peak at $660 \mathrm{~nm}$ ). This device is composed of red LEDs (LXHL-PR09, Luxeon ' III Emitter, Lumileds Lighting, San Jose, CA, USA) uniformly distributed, with a constant power output of $30 \mathrm{~mW} \mathrm{~cm} \mathrm{c}^{-2}$. The $\mathrm{RB}$ sensitizer was illuminated by a white LED device (Fenix ${ }^{\mathrm{rm}}$, LD01, Shanghai, China) with a constant power output of $370 \mathrm{~mW} \mathrm{~cm}^{-2}$.

A Sonidel SP100 sonoporator was used for sonication, at a frequency of $1 \mathrm{MHz}$ and pulse repetition frequency of $100 \mathrm{~Hz}$ (Sonidel Ltd, Dublin, Ireland).

\section{Strain and growth conditions}

C. albicans ATCC 90028 obtained from American Type Culture Collection was used in this study. C. albicans was maintained in yeast peptone glucose medium (YEPD: $1 \%$ yeast extract, $2 \%$ Bacto peptone and 2\% D-glucose, $2 \%$ agar) with glycerol and frozen at $-80^{\circ} \mathrm{C}$ until use. The yeast was subcultured onto Sabouraud dextrose agar plates (SDA) supplemented with chloramphenicol $\left(0.05 \mathrm{~g} \mathrm{l}^{-1}\right)$ and incubated at $37^{\circ} \mathrm{C}$ for $48 \mathrm{~h}$ to grow the colonies used for each experiment. 


\section{Treatment against planktonic cultures}

To prepare the yeast inoculum, five colonies of the agar stock culture were individually transferred to $10 \mathrm{ml}$ of yeast nitrogen base broth (YNB) supplemented with $100 \mathrm{mM}$ glucose, and incubated at $37^{\circ} \mathrm{C}$ overnight. After incubation, $500 \mu \mathrm{l}$ of the culture were transferred to $9.5 \mathrm{ml}$ of fresh YNB and the tube was incubated for $8 \mathrm{~h}$, until $C$. albicans cells reached the mid-log phase of growth. C. albicans suspension was spectrophotometrically standardized $(540 \mathrm{~nm})$ at an OD of 0.7, which corresponds to a final concentration of $10^{7} \mathrm{CFU} \mathrm{ml}{ }^{-1}$. Next, a $100 \mu \mathrm{l}$ aliquot of the inoculum was added to each well of a 96-well polystyrene plate and then submitted to the aPDT or SDT.

Initially, the effect of US application on the viability of planktonic cultures was evaluated. To apply US to the samples, an US gel Rothband (Haslingden, UK) was used to ensure contact between the bottom of the 96-well plate and the US transducer. A wide range of parameters were investigated including duty cycle $(50,55,60,65,70$ and $75 \%)$, exposure time $(2.5,5,7.5$ and $10 \mathrm{~min})$ and power density (intensity) $\left(1,1.5,2,2.5\right.$ and $\left.3 \mathrm{~W} \mathrm{~cm}^{-2}\right)$. To determine yeast survival, aliquots of the contents of each well were serially diluted 10 -fold in sterile saline. Duplicate $25 \mu \mathrm{l}$ aliquots were spread over the surfaces of SDA plates. All plates were aerobically incubated at $37^{\circ} \mathrm{C}$ for $48 \mathrm{~h}$. Then, the colony forming units ( $\left.\mathrm{CFU} \mathrm{ml} \mathrm{m}^{-1}\right)$ were calculated and the effectiveness of aPDT and SDT mediated by $\mathrm{PDZ}$ or RB against the planktonic cultures was evaluated.

For SDT mediated by PDZ, suspensions of C. albicans were incubated with $100 \mu \mathrm{l}$ of the sensitizer solution to yield final PDZ concentrations of 25,50 or $100 \mathrm{mg} \mathrm{l}^{-1}$, and the plates were incubated in the dark for $20 \mathrm{~min}$. Afterwards, the US gel was used between the plate and the transducer of the US device for the US application. US conditions used were a frequency of $1 \mathrm{MHz}$, a power density of $2.5 \mathrm{~W} \mathrm{~cm}^{-2}, 50 \%$ of duty cycle and pulse frequency of $100 \mathrm{~Hz}$ for $5 \mathrm{~min}$ (SDT/PDZ groups). For the RB-mediated SDT, suspensions of $C$. albicans were incubated with $100 \mu \mathrm{l}$ of the sensitizer solution to give final $\mathrm{RB}$ concentrations of 1,5 and $10 \mu \mathrm{M}$, and the plate was incubated in the dark for $30 \mathrm{~min}$ (Costley et al. 2015). Afterwards, the same US parameters as previously described for PDZ were used (SDT/RB groups).

For aPDT mediated by PDZ (aPDT/PDZ groups), $C$. albicans suspensions were incubated for $20 \mathrm{~min}$ (Dovigo et al. 2013) with $100 \mu \mathrm{l}$ of the sensitizer solution to give final PDZ concentrations of 25,50 or $100 \mathrm{mgl}^{-1}$. Then, the plate was irradiated with red LED light $\left(25 \mathrm{~J} \mathrm{~cm}^{-2}\right)$. For aPDT mediated by RB (aPDT/RB groups), suspensions of C. albicans were incubated for $30 \mathrm{~min}$ with $100 \mu \mathrm{l}$ of the sensitizer solution to give final RB concentration of 1,5 and $10 \mu \mathrm{M}$. Then, the samples were irradiated with white LED light $\left(25 \mathrm{~J} \mathrm{~cm}^{-2}\right)$.
For both treatments (SDT and aPDT), additional samples were treated with sensitizer only (PDZ and RB groups), US only (US group), LED light alone (White and Red light groups) or no treatment (Control group). To determine the cell viability after each treatment, the CFU test was performed as described previously. The treatments were performed in duplicate on three separate occasions.

\section{Treatment against biofilms}

For biofilm formation, the yeast suspension was prepared and the concentration adjusted as described previously $\left(10^{7} \mathrm{CFU} \mathrm{ml}{ }^{-1}\right)$. An aliquot of $100 \mu \mathrm{l}$ of the cell suspension was added to the wells of a flat-bottom 96-well polystyrene plate and the culture plate was incubated at $37^{\circ} \mathrm{C}$ in a shaker incubator $(75 \mathrm{rpm}$ ) for $90 \mathrm{~min}$ (adhesion phase). After $90 \mathrm{~min}$, the wells were washed twice with phosphate-buffered saline (PBS) to remove non-adhered cells. Then, $150 \mu \mathrm{l}$ of RPMI 1640 buffered with morpholine propane sulfonic acid (MOPS) (Sigma-Aldrich) at pH 7 were added to each well. After incubation for $48 \mathrm{~h}$ in an orbital shaker (75 rpm) for biofilm formation, the suspension of each well was removed and the biofilm was washed twice with PBS. The biofilms were then treated with either (1) SDT using PDZ or RB, (2) PDT using PDZ or RB and (3) combined PDT/SDT using PDT or RB. Two concentrations of PDZ (175 and $200 \mathrm{mg} \mathrm{l}^{-1}$ ) and RB (100 or $200 \mu \mathrm{M}$ ) were assessed with $100 \mu \mathrm{l}$ of sensitizer added to the biofilm and incubated in the dark for $30 \mathrm{~min}$. Where appropriate, the wells were then treated with light (using a red or white LED at a dose of 50 or $113 \mathrm{~J} \mathrm{~cm}^{-2}$, (PDZ and RB groups respectively) or US (frequency of $1 \mathrm{MHz}$, a power density of $2.5 \mathrm{~W} \mathrm{~cm}^{-2}, 50 \%$ of duty cycle and pulse frequency of $100 \mathrm{~Hz}$ for $5 \mathrm{~min}$ ). For combined aPDT/SDT treatments both light and US were administered simultaneously using the same parameters as described above. After treatment, the biofilm was detached by rubbing the pipette tip for $30 \mathrm{~s}$ in all directions (horizontal, vertical and circular) on the bottom of the well. To determine cell survival in the biofilms, the CFU ml-1 assay was performed in the same way as for the planktonic cultures. The total biomass of biofilm was quantified using crystal violet (CV) staining (Stepanovic et al. 2000; Quishida et al. 2015). After treatments, biofilms were washed with PBS and then fixed with $200 \mu \mathrm{l}$ of methanol for $15 \mathrm{~min}$. The methanol was removed and the plates were kept at $37^{\circ} \mathrm{C}$ for $20 \mathrm{~min}$ to dry. Subsequently, $200 \mu \mathrm{l}$ of CV $\left(1 \% \mathrm{v} \mathrm{v}^{-1}\right)$ were added and maintained for $5 \mathrm{~min}$. The wells were washed with ultrapure water, and after this, 33\% acetic acid was added to remove the dye. The absorbance of the resulting solution at $570 \mathrm{~nm}$ was determined using a microplate. Each evaluation was performed in duplicate on three different occasions. 
The assessment of biofilm morphology and architecture were performed using light and fluorescence microscopy. After treatment, biofilms were washed twice with $0.89 \%$ sterile $\mathrm{NaCl}$. Next, the biofilms were incubated with Trypan Blue solution (TB) (Gibco by Life Technologies, Carlsbad, CA, USA). The stock solution of TB (0.4\%) was diluted in PBS in the proportion of 1:2, respectively. Then, biofilms were incubated with $\mathrm{TB}$ for $15 \mathrm{~min}$, at $37^{\circ} \mathrm{C}$ in the dark. TB was then removed and samples were washed with $0.89 \%$ sterile $\mathrm{NaCl}$ and the fluorophore Concanavalin Alexa Fluor 488 conjugate (Con-A) (Molecular Probes, Inc., Eugene, OR, USA) at a concentration of $100 \mu \mathrm{g} \mathrm{ml}^{-1}$ was added (Chandra et al. 2008). Samples were incubated with Con-A for $30 \mathrm{~min}$ at $37^{\circ} \mathrm{C}$ in the dark and biofilms were then washed with $0.89 \%$ sterile $\mathrm{NaCl}$. The TB solution stains the nucleus of dead cells and the Con-A (excitation wavelength: $488 \mathrm{~nm}$ and emission: $505 \mathrm{~nm}$ long-pass filter) binds to the polysaccharide cell wall with green fluorescence. The TB dye was examined using light microscopy (Leica, London) and the fluorophore Con-A was observed using fluorescent microscopy (Leica), both with a $20 \times$ objective lens. Images were acquired in each microscope and merged using Adobe Photoshop CS6 software (Adobe Systems, San Jose, CA, USA). The thickness of the biofilms was determined using confocal laser scanning microscopy (CLSM) (Zeiss LSM 700 Confocal, Zeiss).

\section{Statistical analysis}

The CFU ml ${ }^{-1}$ values were transformed into $\log _{10}$ and the homogeneity of variance and normality of the data were verified by the Levene and Shapiro-Wilk tests, respectively. The results were analyzed statistically by means of one-way analysis of variance (one-way ANOVA) and, for multiple comparisons, the post hoc Tukey test was applied. The level of significance adopted was $5 \%(\alpha=0.05)$. These analyses were performed using a SPSS software package (IBM ${ }^{\circledR}$ SPSS $^{\circledR}$ Statistics, version 20, Chicago, IL, USA).

\section{Results}

\section{Planktonic culture treatments}

First, the effect of exposing planktonic cultures to US alone was evaluated. The impact of a range of duty cycles, time exposures and power densities were assessed. It was found that exposure to US alone was capable of completely eradicating the $C$. albicans cultures using a duty cycle higher than $50 \%$ at a power density of $3 \mathrm{~W} \mathrm{~cm}^{-2}$, when the US was applied for more than 5 min (data not shown). Therefore, subsequent tests were performed using the US for $5 \mathrm{~min}$, with $50 \%$ of the duty cycle at an US power density of $2.5 \mathrm{~W} \mathrm{~cm}^{-2}$.
Figure 1A shows the results of treatment with SDT mediated by $\operatorname{PDZ}\left(25,50\right.$ or $\left.100 \mathrm{mgl}^{-1}\right)$ or $\mathrm{RB}(1,5,10 \mu \mathrm{M})$, and the control groups (only US, only sensitizer, and control). As shown in Figure 1A, SDT mediated by PDZ 50 (SDT/PDZ 50) or PDZ 100 (SDT/PDZ 100) resulted in eradication of the microorganism. In addition, a significant reduction of $4.35 \log _{10}$ in the SDT/PDZ 25 group was observed $(p=0.001)$. Treatment with US or PDZ separately did not have any significant impact on the viability of $C$. albicans and values were similar $(p \geq 0.984)$ to the control group $\left(6.38 \log _{10}\right)$. Treatment with SDT mediated by $\mathrm{RB} 5$ (SDT/RB 5) or RB 10 (SDT/RB 10) resulted in the eradication of the microorganism. In addition, it a significant reduction $(p=0.001)$ of $5.01 \log _{10}$ was observed in the group treated with SDT mediated by RB 1 (SDT/RB 1) when compared with the untreated control group (Figure 1B). The results also demonstrated that when cells were treated with $\mathrm{RB}$ alone at concentrations of 5 and $10 \mu \mathrm{M}$ the viability of planktonic cultures decreased significantly $(p \leq 0.028)$ (Figure 1B).

The aPDT mediated by PDZ 100 (aPDT/PDZ 100) resulted in the eradication of the microorganism. A significant reduction $(p \leq 0.001)$ in cell viability was also observed in the aPDT/PDZ 25 and aPDT/PDZ 50 treatment groups equivalent to 5.23 and $5.87 \log _{10}$, respectively, compared with the control group. Treatment with red LED light or PDZ alone had no effect on the viability of $C$. albicans and the results were similar to the control group ( $p>=0.989)$ (Figure 1C).

The aPDT mediated by RB 10 (aPDT/RB 10) resulted in the eradication of $C$. albicans. In addition, compared with the control group, a significant reduction $(p=0.001)$ in viability of the groups treated with $\mathrm{RB} 1$ (aPDT/RB 1) and RB 5 (aPDT/RB 5) of 5.24 and $5.39 \log _{10}$, respectively, was achieved. The use of light alone did not affect the microorganism and it was similar to the control group $(p=0.999)$ (Figure 1D).

\section{Biofilm treatments}

The effect of US treatment alone on biofilms was also evaluated, testing a range of treatment times $(5,6,7,8$ and $10 \mathrm{~min}$ ) and US power densities $\left(2.5\right.$ and $\left.3 \mathrm{~W} \mathrm{~cm}^{-2}\right)$. It was observed that US in the absence of a sensitizer reduced the viability of C. albicans biofilms after application for $6 \mathrm{~min}$ at power densities of 2.5 or $3 \mathrm{~W} \mathrm{~cm}^{-2}$ (data not shown). For this reason, the subsequent tests were performed using the US for $5 \mathrm{~min}$, with $50 \%$ of duty cycle at $2.5 \mathrm{~W} \mathrm{~cm}^{-2}$.

The association of aPDT + SDT mediated by PDZ at the concentrations of 175 or $200 \mathrm{mg} \mathrm{l}^{-1}$ resulted in significant $(p \leq 0.001)$ reductions in viability of 2.08 and $3.39 \log _{10}$, respectively (Figure $2 \mathrm{~A}$ ). Moreover, the combination of 

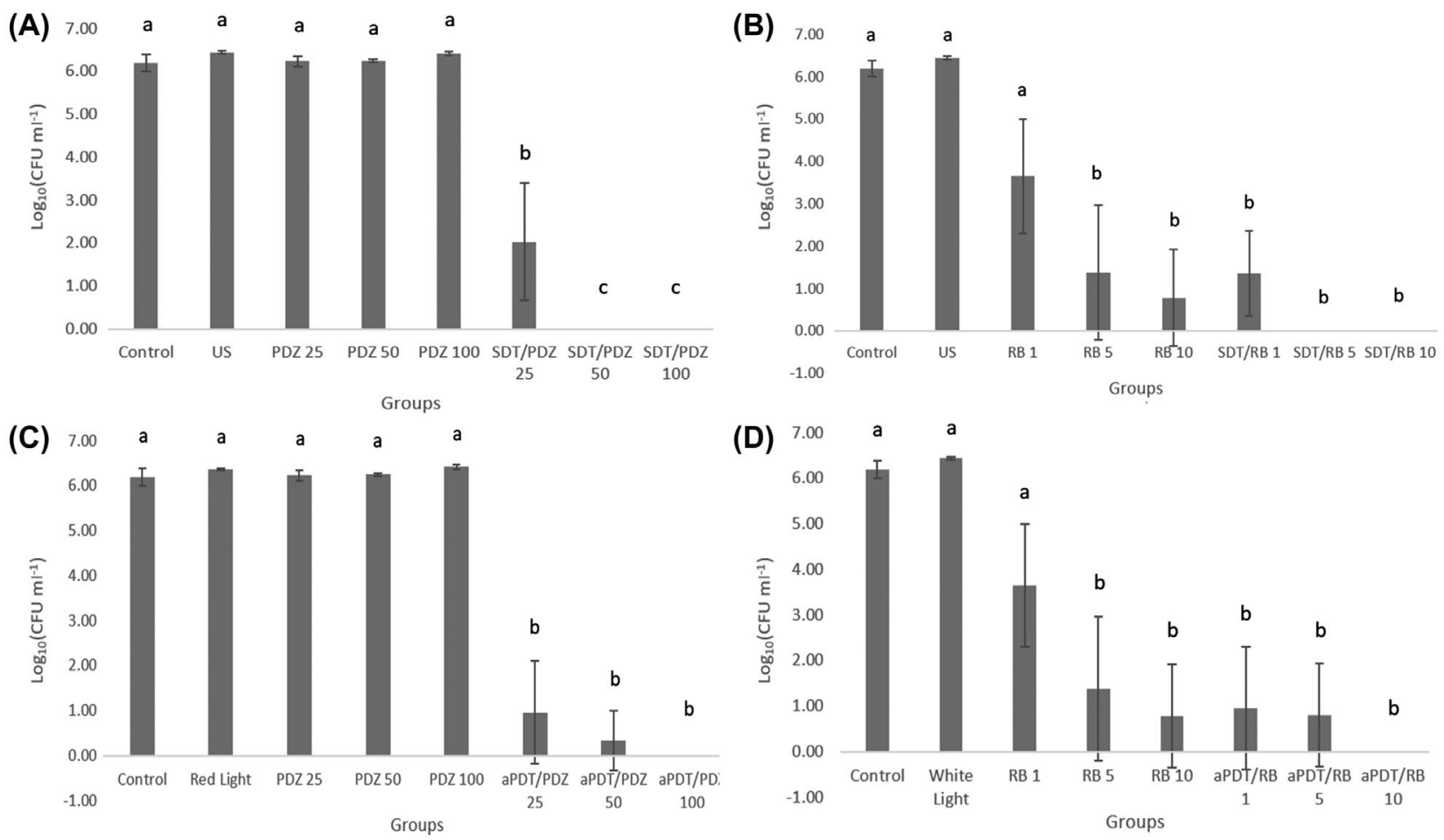

Figure 1. (A, B) Mean values and SD of $\log _{10}($ CFU ml-1) of planktonic cultures of C. albicans treated with SDT mediated by PDZ (SDT/ PDZ 25, SDT/PDZ 50, SDT/PDZ 100 groups) or RB (SDT/RB 1, SDT/RB 5, and SDT/RB 10 groups), and control groups: control, only US (US group) and only sensitizer (PDZ 25, PDZ 50, PDZ 100, RB 1, RB 5 and RB 10 groups). US: 5 min, $50 \%$ of duty cycle, 2.5 W cm ${ }^{-2}$. (C, D) Mean values and SD of $\log _{10}\left(\mathrm{CFU} \mathrm{ml} \mathrm{m}^{-1}\right.$ ) of planktonic cultures of $C$. albicans treated with aPDT mediated by PDZ (aPDT/PDZ 25, aPDT/PDZ 50 and aPDT/PDZ 100 groups) or RB (aPDT/RB 1, aPDT/RB 5, and aPDT/RB 10 groups), and control groups: control, only light (Red or White light groups) and only sensitizer (PDZ 25, PDZ 50, PDZ 100, RB 1, RB 5 and RB 10 groups). Red light: LED 660 nm, 25 J cm-2. White light: white LED, $25 \mathrm{~J} \mathrm{~cm}^{-2}$. Equal letters denote statistical similarity $(p>0.05)$.

aPDT + SDT mediated by RB at concentrations of 100 or $200 \mu \mathrm{M}$ resulted in significant $(p \leq 0.001)$ reductions in viability equivalent to 1.45 and $1.91 \log _{10}$, respectively (Figure 2B). The treatment with only aPDT or SDT did not reduce the viability of $C$. albicans and the results were similar to the control group ( $p=1.000)$ (Figure $2 \mathrm{~A}$ and $\mathrm{B}$ ). The viability of the microorganism after treatment with PDZ, RB or Light+US separately was also similar to the control group $(p \geq 0.999)$ (Figure 2A and B).

The CV assay demonstrated that the combined treatment aPDT+SDT resulted in a significant reduction in the total biomass. The aPDT+SDT mediated by PDZ at 175 or $200 \mathrm{mg} \mathrm{l}^{-1}$ resulted in a significant reduction $(p \leq 0.001)$ of 75.5 and $71.2 \%$, respectively, compared to the control group (Figure $2 \mathrm{C}$ ). The treatment with aPDT+SDT mediated by RB at 100 or $200 \mu \mathrm{M}$ was able to significantly reduce $(p \leq 0.001)$ the total biomass by 62.1 and $73.0 \%$, respectively (Figure 2D). On the other hand, only the application of aPDT mediated by PDZ 200 (aPDT/PDZ $200)$ significantly reduced the total biomass by $~ 30.9 \%$ $(p=0.025)$ (Figure 2C). The aPDT mediated by RB at 100 and 200 (aPDT/RB 100 and aPDT/RB 200 groups) was able to significantly $(p \leq 0.012)$ reduce the total biomass by 34.8 and $35.2 \%$, respectively (Figure $2 \mathrm{D}$ ). The total biomass of the biofilms treated with SDT mediated by PDZ was similar to the control group $(p \geq 0.823)$ (Figure $2 \mathrm{C})$. On the other hand, the RB-mediated SDT significantly $(p \leq 0.005)$ reduced the total biomass of the biofilms by 36.6 and $53.4 \%$ when compared to the control group (Figure 2D).

The assessment of biofilm morphology and architecture was performed using light and fluorescence microscopy (Figure 3). The image captured in the control group shows a denser biofilm, a high concentration of live cells (blastopore and hypha) and a thickness equivalent to $88.54 \mu \mathrm{m}$ when measured by CLSM. The biofilms treated with aPDT+SDT mediated by 175 or $200 \mathrm{mg} \mathrm{l}^{-1}$ of PDZ showed a reduction in biofilm density with an equivalent thickness of 45.3 and $44.6 \mu \mathrm{m}$, respectively, a smaller number of cells, a reduction in the filamentous form and most of the cell nuclei were stained with TB, indicating cell death. The images obtained in the groups treated with aPDT+SDT mediated by 100 or $200 \mathrm{mg} \mathrm{l}^{-1}$ of RB demonstrated a reduced density (with a thickness equivalent 42.07 and $49.84 \mu \mathrm{m}$, respectively), a reduction in hyphae and most of the cell nuclei had stained with TB. The results visualized by microscopy are in accordance with the results obtained in the CFU and total biomass 

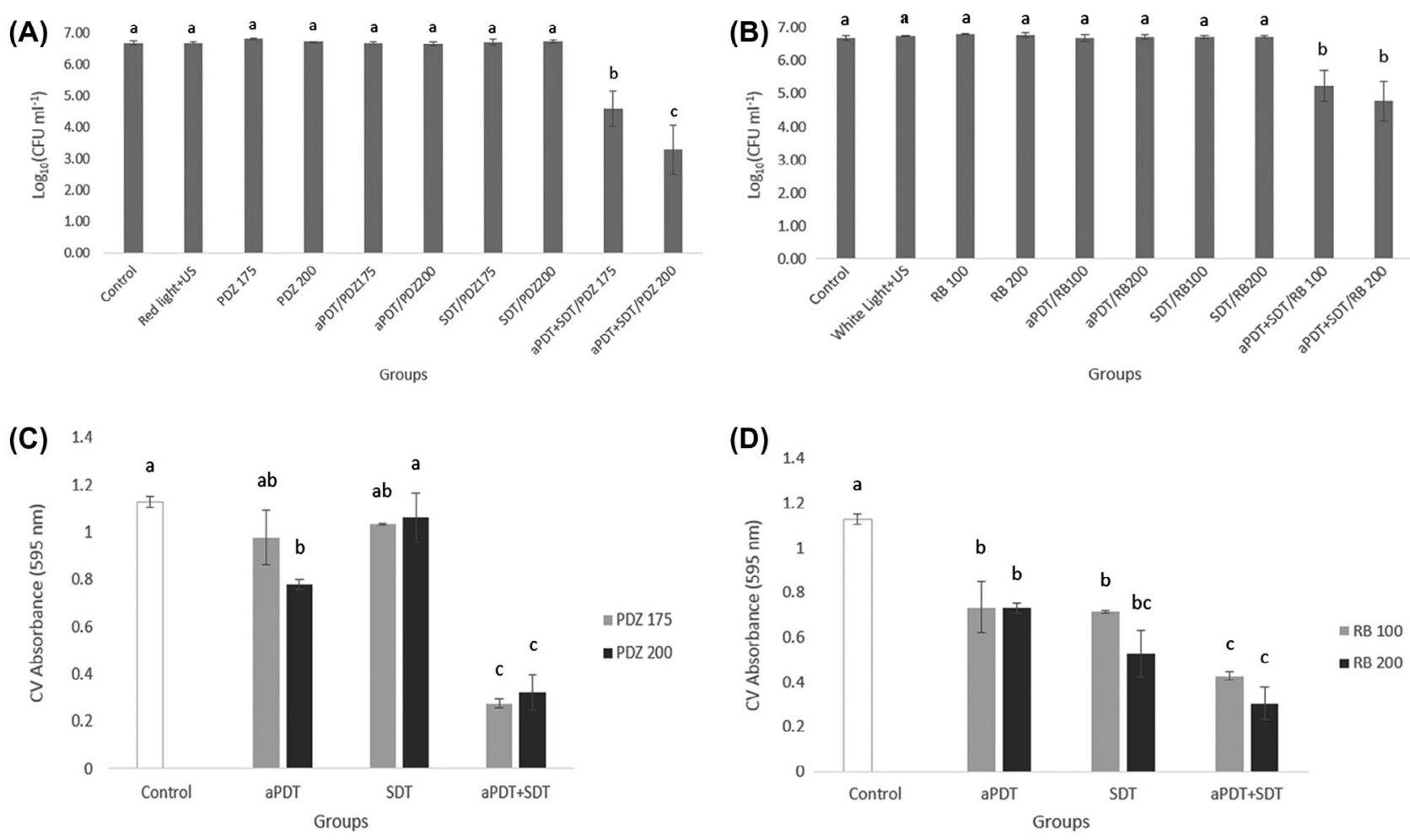

Figure 2. (A, B) Mean values and SD of $\log _{10}\left(C F U \mathrm{ml}^{-1}\right)$ of $C$. albicans biofilms treated with aPDT, SDT, aPDT+SDT mediated by PDZ (175 and $200 \mathrm{mg} \mathrm{l}^{-1}$ ) or RB (100 or $200 \mu \mathrm{M}$ of RB) and control groups (Control, Red light+US, White light+US, PDZ 175, PDZ 200, RB 100 and RB 200 groups). Red light: LED $660 \mathrm{~nm}, 50 \mathrm{~J} \mathrm{~cm}^{-2}$. White light: $113 \mathrm{~J} \mathrm{~cm}^{-2}$. US: $5 \mathrm{~min}, 50 \%$ of duty cycle, $2.5 \mathrm{~W} \mathrm{~cm}{ }^{-2}$. (C, D) Mean values and SD of crystal violet absorbance of C. albicans biofilms treated with aPDT, SDT and aPDT+SDT mediated by PDZ (175 or $200 \mathrm{mg} \mathrm{I}^{-1}$ ) or RB (100 or $200 \mu \mathrm{M})$. Red light: $50 \mathrm{~J} \mathrm{~cm}^{-2}$. White light: $113 \mathrm{~J} \mathrm{~cm}^{-2}$. US: $5 \mathrm{~min}, 50 \%$ of duty cycle, $2.5 \mathrm{~W} \mathrm{~cm} \mathrm{~cm}^{-2}$. Equal letters denote statistical similarity $(p>0.05)$.

assays, where the combined treatment of SDT and aPDT reduced significantly the viability of $C$. albicans.

\section{Discussion}

To overcome the problems associated with antifungal resistance, studies have been performed to search for alternative therapies to treat infections. aPDT (Dovigo, Pavarina, Carmello et al. 2011; Dovigo et al. 2013; Pereira et al. 2011; Costa et al. 2012) and SDT (Ma et al. 2009; Wang et al. 2014; Zhuang et al. 2014; Costley et al. 2015; Serpe and Giuntini 2015) have been suggested as promising therapies for the inactivation of microorganisms. According to the data, it was observed that the application of aPDT, mediated by PDZ at $100 \mathrm{mg} \mathrm{l}^{-1}$ or RB $10 \mu \mathrm{M}$, eradicated planktonic cultures of $C$. albicans. These results agree with those found by Dovigo et al. (2013) and Freire et al. (2014) who observed complete killing of C. albicans suspensions, when aPDT was mediated by PDZ (50 and $\left.75 \mathrm{mg}^{-1}\right)$ and $\mathrm{RB}(12.5 \mu \mathrm{M})$, respectively. On the other hand, other studies assessing RB as PS to mediate aPDT obtained different results. Costa et al. (2012) observed that $\mathrm{RB}$ at $40 \mu \mathrm{M}$ against clinical isolates of $C$. albicans resulted in a reduction equivalent to $1.97 \log _{10}$. In another investigation, using RB at $200 \mu \mathrm{mol} \mathrm{l}^{-1}$ for $20 \mathrm{~min}$, reductions of 4 $\log _{10}$ and $6 \log _{10}$ were observed for cellular densities of $10^{7}$ and $10^{6}$ cells $\mathrm{ml}^{-1}$, respectively (Demidova and Hamblin 2005). The divergent results obtained may be attributed to the different aPDT parameters and type of strains (clinical isolates $\times$ reference strains) used.

With respect to the SDT results in planktonic cultures, this treatment was also able to eradicate the microorganism. To the best of the authors' knowledge, this is the first study that aimed to assess SDT for the inactivation of $C$. albicans. The effectiveness of this treatment has been demonstrated against pathogenic bacterial species (Kremkau et al. 1976; Nakonechny et al. 2013; Wang et al. 2014; Zhuang et al. 2014; Costley et al. 2015). The results of these studies and those found in the present investigation demonstrate that SDT is able to inactivate pathogenic microbial species, including C. albicans, when cultivated in suspensions.

The extracellular matrix of the biofilm that surrounds the microbial cells can act as a physical barrier (Ramage et al. 2009). For this reason, the organization of C. albicans as a biofilm is a significant challenge associated with the 
TB
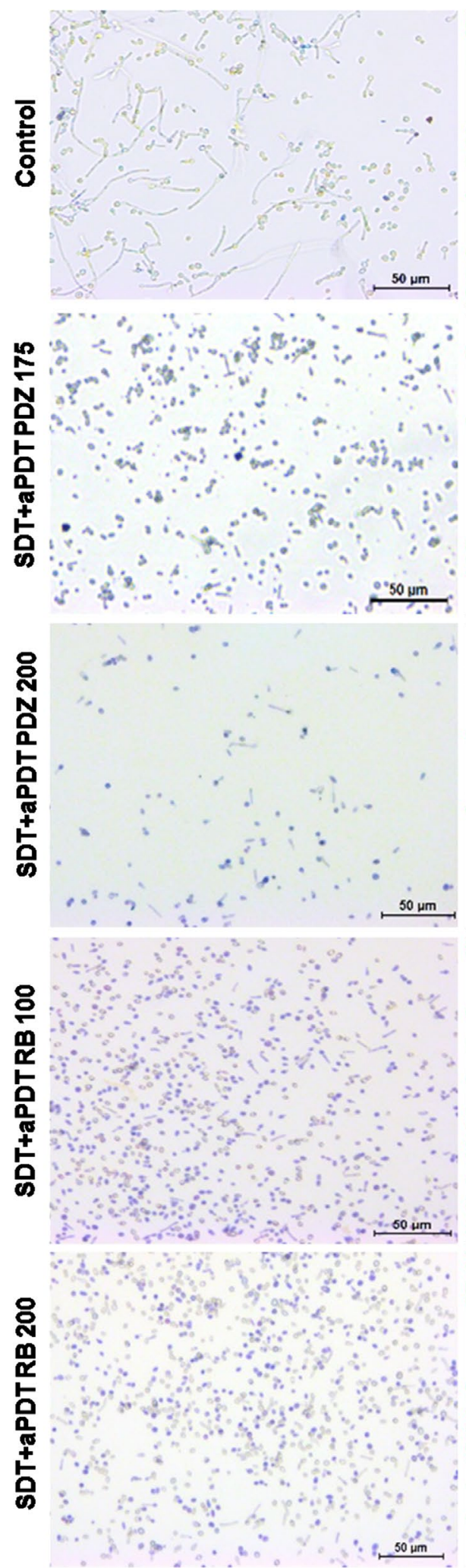

Con-A
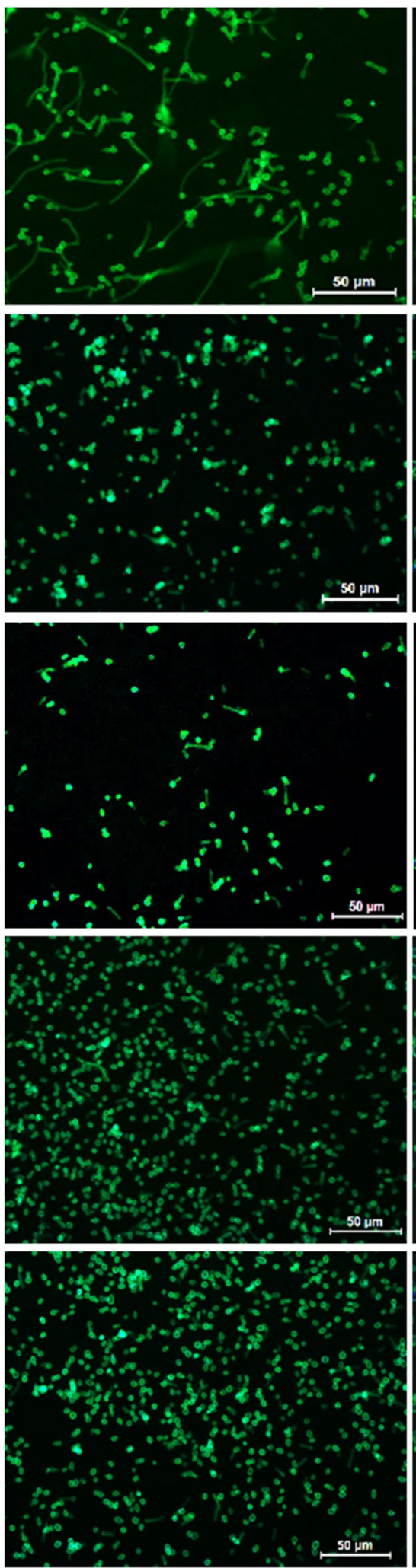

Merged detailed
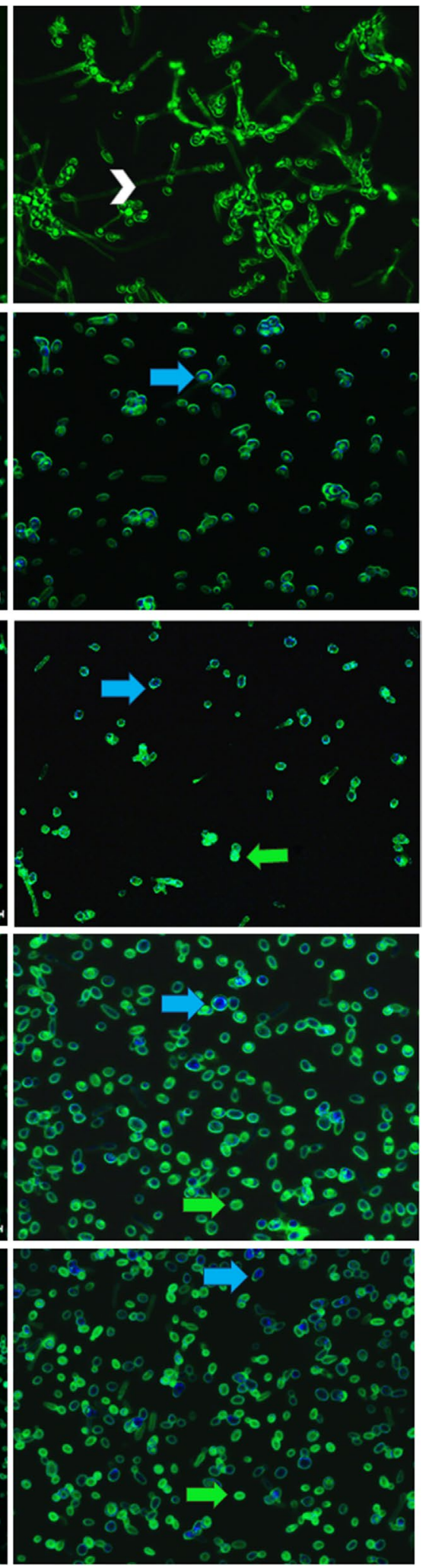

Figure 3. Light and fluorescence microscope images of the Control group and biofilms treated with aPDT+SDT. Samples were stained with Trypan Blue and Con-A. The TB solution stained the nuclei of dead cells (blue arrow) and the Con-A bound to the polysaccharide cell wall with green fluorescence (green arrow). The TB dye was examined under a light microscope ('TB' column) and Con-A was observed under a fluorescent microscope ('Con- $A$ ' column) (magnification 20X). The'Merged detailed' column shows in detail the images that were captured in the light and fluorescent microscope and, then, merged. Arrow head: hyphae cell. 
uptake of the sensitizer. In the present investigation, it was found that the simultaneous application of aPDT+SDT produced an enhanced effect compared to each treatment alone (SDT or aPDT). Significant reductions of 3.39 and $1.91 \log _{10}$ were observed when the highest concentration of PDZ and RB were used, respectively. Some authors hypothesize that this increase may be a result of a synergistic aPDT and SDT effect due to US-induced diffusion of the sensitizers. Costley et al. (2015) proved that the pre-treatment of Pseudomonas aeruginosa biofilm with US for 5 min before the addition of RB produced a 2.6fold increase in sensitizer diffusion through the biofilm compared with the untreated biofilm control. According to the authors, the US facilitated dispersion of sensitizers through the biofilms, thereby improving efficacy of the treatment (Costley et al. 2015). It has also been suggested that US increases the uptake of molecules through transient pores formed in the membrane (Bao et al. 1997; Miller 2000; Rosenthal et al. 2004), a process known as sonoporation. During this process, exogenous molecules, such as sensitizers, can enter into the cells through these pores, which can reseal after the uptake (Bao et al. 1997; Miller 2000; Rosenthal et al. 2004). Another possibility is that the physical agitation of the solution from the applied US causes circulation of the microorganism in the wells and increases exposure to the light source. Possibly one treatment must be sensitizing the fungal cells making the other more effective. However, to gain a more comprehensive understanding of the mechanisms involved and possible effects further study is required.

Regarding the application of aPDT or SDT separately, it was observed that these treatments were not effective in reducing the viability of $C$. albicans biofilm. It has been demonstrated that Candida biofilms are less susceptible to aPDT when compared with their planktonic counterparts (Dovigo, Pavarina, Mima et al. 2011; Dovigo et al. 2013; Costa et al. 2012) and in the present investigation it was also observed for the aPDT and SDT treatments. Previous studies have shown that aPDT mediated by PDZ or RB reduced the viability of $C$. albicans biofilm cells by $<1 \log _{10} 1$ (Dovigo, Pavarina, Mima et al. 2011, Dovigo et al. 2013; Costa et al. 2012). The aPDT mediated by PDZ (125 $\left.\mathrm{mg} \mathrm{l}^{-1}\right)$ and LED light $\left(37.5 \mathrm{~J} \mathrm{~cm}^{-2}\right)$ resulted in a reduction in C. albicans viability by $0.9 \log _{10}$ in biofilms (clinical isolates) (Dovigo et al. 2013). The aPDT mediated by RB $\left(40 \mu \mathrm{mol} \mathrm{l}^{-1}\right)$ promoted a reduction in viability of $0.12 \log _{10}$ in clinical isolates of $C$. albicans grown as biofilms (Costa et al. 2012). In another study, aPDT using $200 \mu \mathrm{mol} \mathrm{l}^{-1}$ of RB reduced viability by $0.22 \log _{10}$ in C. albicans biofilm (Freire et al. 2014). As this was the first study that aimed to assess SDT for the inactivation of fungal biofilms, it is not possible to directly compare the results found in this investigation.
The treatments employed here were also evaluated through quantification of total biomass by CV staining. The treatments performed simultaneously (aPDT+SDT) showed enhanced results in the CV assay. The highest concentrations of the sensitizers were able to reduce the total biomass of the biofilm by 71.2 and $73.0 \%$ when PDZ and $\mathrm{RB}$ were used, respectively. It is important to emphasize that the CV stains the matrix as well as both living and dead cells within the biofilm (Peeters et al. 2008). It is possible to hypothesize that the combination of both treatments was able to disrupt the biofilm, enabling penetration of the sensitizer more deeply into the biofilm. In addition, as mentioned previously, US can increase the uptake of the sensitizer via sonoporation (Bao et al. 1997; Miller 2000). Once the sensitizer penetrates more deeply into the biofilm and is taken up by the microorganisms, the light and US will then activate the sensitizer, resulting in cell death. For this reason, it is suggested that the possible ability of the combined treatment to disrupt the biofilm is important for the dispersion, uptake and activation of the sensitizer and consequently, for the effectiveness of the treatment.

When aPDT was mediated by $\mathrm{RB}$, a significant reduction in the total biomass by up to $35.2 \%$ was observed, depending on the concentration of the sensitizer. The application of aPDT mediated by PDZ at $200 \mathrm{mg} \mathrm{l}^{-1}$ reduced the biomass of the biofilm by $30.9 \%$. These results are in agreement with those found in the literature which observed that the combination of $150 \mathrm{mg} \mathrm{l}^{-1}$ of PDZ associated with LED light produced reductions on total biomass equivalent to $24.4,39.2$ and $43.7 \%$ for C. albicans, Candida tropicalis and C. glabrata, respectively (Carmello et al. 2017). The total biomass of the biofilms treated with SDT mediated by PDZ was similar to the control group. On the other hand, the RB-mediated SDT significantly reduced the total biomass of the biofilms. As stated above, this is the first study that aimed to assess the effect of SDT against biofilms and therefore it is not possible to compare directly the $\mathrm{CV}$ results found in the present investigation.

The biofilm morphology and architecture after the application of the combined treatment $(\mathrm{aPDT}+\mathrm{SDT})$ was assessed using light and fluorescence microscopy. The images revealed that the biofilms treated with aPDT + SDT, mediated by PDZ and RB, showed a thinner thickness, a smaller number of cells, a reduction in the filamentous form and increased cell death. These results show that this new approach reduced the viability, disrupted the integrity of the biofilm and eliminated most of the filamentous cells. The fact that this treatment is able to reduce the filamentous form is a relevant finding. The ability of $C$. albicans to switch from unicellular yeast into filamentous form is thought to be important for Candida 
virulence (Thompson et al. 2011). This type of morphology can invade layers of epithelial cells, to exert mechanical force, promote penetration and growth between the epithelial cells (Thompson et al. 2011). Thus, the filamentous form facilitates the invasion of the host tissue. The study conducted by Quishida et al. (2015) evaluated the architecture of mixed biofilms formed by C. albicans, $C$. glabrata and Streptococcus mutans that were submitted to aPDT mediated by PDZ using CLSM. According to the authors, a visual increase in dead cells was observed in the biofilms incubated with PDZ at $175 \mathrm{mg} \mathrm{ml}^{-1}$ and irradiated with $37.5 \mathrm{~J} \mathrm{~cm}^{-2}$. However, this treatment did not reduce the numbers of cells, the thickness of the treated biofilm was similar to the control group and there was no change in cell morphology (Quishida et al. 2015).

The US power density (intensity), frequency, and exposure time have been shown to be important parameters for SDT antimicrobial effects (Nakonechny et al. 2013; Wang et al. 2014; Zhuang et al. 2014; Costley et al. 2015) and this was also observed in the present investigation. This study showed that US, when applied in the absence of sensitizer, was able to reduce Candida survival in planktonic cultures and biofilms when delivered at power densities $>2.5 \mathrm{~W} \mathrm{~cm}^{-2}, 50 \%$ of DC and exposure for $5 \mathrm{~min}$. The killing of C. albicans may be attributed to an US-induced increase in temperature (Kremkau et al. 1976; Harrison and Balcer-Kubiczek 1991). Thermal effects produced by US have been used for other therapeutic applications. The US-induced tissue hyperthermia, which transiently increases the tissue temperature to $40-45^{\circ} \mathrm{C}$, has been studied for clinical benefits such as inflammation relief, physical therapy and enhancement of cancer chemotherapy. For thermal ablation, US can be used to increase tissue temperature to $60-85^{\circ} \mathrm{C}$ and has been used for non-invasive surgery in the treatment of solid tumors, sealing of blood vessels and correction of cardiac arrhythmias (Rosenthal et al. 2004). It is important to emphasize that, exposure to the US for $5 \mathrm{~min}$, with $50 \%$ of duty cycle at an US power density of $2.5 \mathrm{~W} \mathrm{~cm}^{-2}$ did not affect the viability of C. albicans (suspension and biofilm). Therefore, subsequent tests evaluating the effectiveness of SDT and aPDT+SDT were performed using these parameters. For this reason, it is concluded that the effect of aPDT+SDT against $C$. albicans was a result of the treatment and not to the increase in temperature. Therefore, the determination of appropriate US parameters is crucial for using the US for antimicrobial proposes.

In addition, it was observed that the treatment with RB sensitizer in the absence of US or light reduced $C$. albicans survival in suspension. The study conducted by Maliszewska et al. (2017) verified that RB at concentrations $>25 \mathrm{mg} \mathrm{l}^{-1}$ was able to significantly reduce planktonic cultures of C. albicans (ATCC 10231, $10^{5} \mathrm{cells} \mathrm{ml}^{-1}$ ).
On the other hand, Freire et al. (2014) observed that RB in concentrations ranging from 0.78 to $200 \mu \mathrm{M}$ had no effect on C. albicans suspensions. However, these authors evaluated another reference strain of $C$. albicans (ATCC 18804), the inoculum was cultured in different medium (Sabouraud dextrose broth) and the C. albicans suspension was incubated for only 5 min with RB. In contrast, when PDZ was applied in the absence of light or US, this PS was not able to reduce the suspension viability of $C$. albicans. The non-toxicity of PDZ has been reported in the literature (Dovigo et al. 2013; Carmello et al. 2017). Therefore, the effect of the sensitizers on the C. albicans viability depends on the type of the PS evaluated.

The present investigation also demonstrated that the treatments mediated by the sensitizer PDZ were more effective on both planktonic and biofilm phase growths in comparison with the $\mathrm{RB}$ treatments. These results obtained may be attributed to the different characteristics of each sensitizer. The sensitizer PDZ, classified as a second-generation PS, is obtained from the cyanobacterium Spirulina platensis as a noncovalent complex of $\mathrm{N}$-methyl-D-glucosamine chlorine e6 salt on basis of chlorophyll $a$ derivatives. PDZ has an absorption peak in the red region of the spectrum $(660 \mathrm{~nm})$ and it is known to produce a high amount of singlet oxygen (Ferreira et al. 2008). On the other hand, the xanthene dye RB is an anionic water-soluble synthetic fluorescein derivative, which has an absorption peak in the green region of the spectrum (450-600 $\mathrm{nm}$ ) and a low rate of photodegradation (Spagnul et al. 2015). This PS is capable of photo-catalytic conversion of an oxygen molecule to singlet oxygen under $532 \mathrm{~nm}$ light irradiation, with a singlet oxygen quantum yield of $\sim 76 \%$ (Encinas et al. 2009). Moreover, the higher efficacy of the aPDT treatment mediated by PDZ in comparison with RB may also be attributed to the wavelength of the LED device used. PDZ was excited by a LED device with the appropriated wavelength for this sensitizer $(660 \mathrm{~nm})$ and RB was not, and this may influence the efficacy of the treatment.

In conclusion, the results described above demonstrate the potential of combined aPDT/aSDT treatment as an alternative approach for the eradication of fungal biofilms. A powerful synergy was observed when the biofilms were treated with both aPDT and aSDT compared to either treatment alone. The reason for this synergy remains unknown but may result from SDT sensitizing the biofilm to PDT treatment. As mentioned previously, US is known to facilitate the dispersion of drugs through impermeable tissue as a result of cavitation induced effects. Therefore, SDT treatment may facilitate the generation of transient pores in the biofilm matrix enabling a greater diffusion of both the sensitizer and light. The authors are currently exploring this hypothesis in greater detail. In addition, they 
are also interested in exploring the effects of the treatment on biofilms in vivo and its effect on the host-tissue. Given the issues associated with multi-drug resistant infections it is crucial that new approaches to treat such conditions are developed soon. It is believed that combined aPDT/SDT treatment could be one such approach for the targeted and efficacious control of localized fungal infection.

\section{Acknowledgements}

We thank Paula Aboud Barbugli and Bridgeen Callan for the help with the microscopy images.

\section{Disclosure statement}

The authors declare that they have no conflict of interest.

\section{Funding}

This work was supported by the São Paulo Research Foundation (FAPESP) [grant numbers 2016/00190-2, 2014/01262-1, 2013/ 07276-1] and PROPG-UNESP [grant 03/2016].

\section{ORCID}

Ana Cláudia Pavarina (D) http://orcid.org/0000-0002-92311994

\section{References}

Banting DW, Greenhorn PA, McMinn JG. 1995. Effectiveness of a topical antifungal regimen for the treatment of oral candidiasis in older, chronically ill, institutionalized, adults. J Can Dent Assoc. 61:199-200, 203-205.

Bao S, Thrall BD, Miller DL. 1997. Transfection of a reporter plasmid into cultured cells by sonoporation in vitro. Ultrasound Med Biol. 23:953-959.. doi:10.1016/S03015629(97)00025-2

Barchiesi F, Arzeni D, Del Prete MS, Sinicco A, Falconi Di Francesco L, Pasticci MB, Lamura L, Nuzzo MM, Burzacchini F, Coppola S, et al. 1998. Fluconazole susceptibility and strain variation of Candida albicans isolates from HIV-infected patients with oropharyngeal candidosis. J Antimicrob Chemother. 41:541-548. Erratum in: J Antimicrob Chemother 1998; 42:413. doi:10.1093/ jac/41.5.541

Bliss JM, Bigelow CE, Foster TH, Haidaris CG. 2004. Susceptibility of Candida species to photodynamic effects of Photofrin. Antimicrob Agents Chemother. 48:2000-2006. doi:10.1128/AAC.48.6.2000-2006.2004

Bonnett R, Martínez G. 2001. Photobleaching of sensitisers used in photodynamic therapy. Tetrahedron. 57:9513-9547. doi:10.1016/S0040-4020(01)00952-8

Carmello JC, Alves F, Mima EG, Jorge JH, Bagnato VS, Pavarina AC. 2017. Photoinactivation of single and mixed biofilms of Candida albicans and non-albicans Candida species using Phorodithazine". Photodiagnosis Photodyn Ther. 17:194-199. Erratum in: Photodiagnosis Photodyn Ther. 2017; 20:288. doi:10.1016/j.pdpdt.2016.11.013
Chandra J, Mukherjee PK, Ghannoum MA. 2008. In vitro growth and analysis of Candida biofilms. Nat Protoc. 3:1909-1924. doi:10.1038/nprot.2008.192

Costa AC, Rasteiro VM, Pereira CA, Rossoni RD, Junqueira JC, Jorge AO. 2012. The effects of rose bengal- and erythrosinemediated photodynamic therapy on Candida albicans. Mycoses. 55:56-63. doi:10.1111/myc.2011.55.issue-1

Costerton JW, Stewart PS, Greenberg EP. 1999. Bacterial biofilms: a common cause of persistent infections. Science. 284:1318-1322. doi:10.1126/science.284.5418.1318

Costley D, Mc Ewan C, Fowley C, McHale AP, Atchison J, Nomikou N, Callan JF. 2015. Treating cancer with sonodynamic therapy: a review. Int J Hyperthermia. 31:107117. doi:10.3109/02656736.2014.992484

Costley D, Nesbitt H, Ternan N, Dooley J, Huang YY, Hamblin MR, McHale AP, Callan JF. 2017. Sonodynamic inactivation of Gram-positive and Gram-negative bacteria using a Rose Bengal-antimicrobial peptide conjugate. Int J Antimicrob Agents. 49:31-36. doi:10.1016/j.ijantimicag.2016.09.034

Demidova TN, Hamblin MR. 2005. Effect of cell-photosensitiser binding and cell density on microbial photoinactivation. Antimicrob Agents Chemother. 49:2329-2335. doi:10.1128/ AAC.49.6.2329-2335.2005

Donnelly RF, McCarron PA, Tunney MM. 2008. Antifungal photodynamic therapy. Microbiol Res. 163:1-12. doi:10.1016/j.micres.2007.08.001

Dovigo LN, Carmello JC, Carvalho MT, Mima EG, Vergani CE, Bagnato VS, Pavarina AC. 2013. Photodynamic inactivation of clinical isolates of Candida using Photodithazine ${ }^{\bowtie}$. Biofouling. 29:1057-1067. doi:10.1080/08927014.2013.827668

Dovigo LN, Pavarina AC, Carmello JC, Machado AL, Brunetti IL, Bagnato VS. 2011. Susceptibility of clinical isolates of Candida to photodynamic effects of curcumin. Lasers Surg Med. 43:927-934. doi:10.1002/lsm.v43.9

Dovigo LN, Pavarina AC, Mima EGO, Giampaolo ET, Vergani CE, Bagnato VS. 2011. Fungicidal effect of photodynamic therapy against fluconazole-resistant Candida albicans and Candida glabrata. Mycoses. 54:123-130. doi:10.1111/ myc.2011.54.issue-2

Ellepola AN, Samaranayake LP. 2000. Antimycotic agents in oral candidosis: an overview: 2. Treatment of oral candidosis. Dent Update. 27:165-170, 172-174. doi: 10.12968/denu.2000.27.4.165

Encinas MC, Rufs AM, Bertolotti SG, Previtali CM. 2009. Xanthene dyes/amine as photoinitiators of radical polymerization: a comparative and photochemical study in aqueous medium. Polymer. 50:2762-2767. doi:10.1016/j. polymer.2009.04.024

Ferreira J, Menezes PFC, Kurachi C, Sibata C, Allison RR, Bagnato VS. 2008. Photostability of different chlorine photossensitisers. Laser Phys Lett. 5:156-161. doi:10.1002/ lapl.200710099

Flemming HC, Wingender J. 2010. The biofilm matrix. Nat Rev Microbiol. 8:623-633. doi:10.1038/nrmicro2415

Freire F, Costa AC, Pereira CA, Beltrame Junior M, Junqueira JC, Jorge AO. 2014. Comparison of the effect of rose bengaland eosin Y-mediated photodynamic inactivation on planktonic cells and biofilms of Candida albicans. Lasers Med Sci. 29:949-955. doi:10.1007/s10103-013-1435-x

Goldman GH, da Silva Ferreira ME, dos Reis Marques Everaldo, Savoldi M, Perlin D, Park S, Godoy Martinez PC, Goldman MH, Colombo AL. 2004. Evaluation of fluconazole resistance mechanisms in Candida albicans 
clinical isolates from $\mathrm{HIV}$-infected patients in Brazil. Diagn Microbiol Infect Dis. 50:25-32. doi:10.1016/j. diagmicrobio.2004.04.009

Harrison GH, Balcer-Kubiczek EK. 1991. Pulsed ultrasound and neoplastic transformation in vitro. Ultrasound Med Biol. 17:627-632. doi:10.1016/0301-5629(91)90033-S

Hiraoka W, Honda H, Feril LB Jr, Kudo N, Kondo T. 2006. Comparison between sonodynamic effect and photodynamic effect with photosensitisers on free radical formation and cell killing. Ultrason Sonochem. 13:535-542. doi:10.1016/j.ultsonch.2005.10.001

Hunter KD, Gibson J, Lockhart P, Pithie A, Bagg J. 1998. Fluconazole-resistant Candida species in the oral flora of fluconazole-exposed HIV-positive patients. Oral Surg Oral Med Oral Pathol Oral Radiol Endod. 85:558-564. doi:10.1016/S1079-2104(98)90291-8

Kremkau FW, Kaufmann JS, Walker MM, Burch PG, Spurr CL. 1976. Ultrasonic enhancement of nitrogen mustard cytotoxicity in mouse leukemia. Cancer. 37:1643-1647. doi:10.1002/(ISSN)1097-0142

Kulak Y, Arikan A, Delibalta N. 1994. Comparison of three different treatment methods for generalized denture stomatitis. J Prosthet Dent. 72:283-288. doi:10.1016/00223913(94)90341-7

Lombardi T, Budtz-Jorgensen E. 1993. Treatment of denture induced stomatitis: a review. Eur J Prosthodont Restor Dent. 2:17-22.

Ma X, Pan H, Wu G, Yang Z, Yi J. 2009. Ultrasound may be exploited for the treatment of microbial diseases. Med Hypotheses. 73:18-19. doi:10.1016/j.mehy.2009.01.033

Maliszewska I, Lisiak B, Popko K, Matczyszyn K. 2017. Enhancement of the efficacy of photodynamic inactivation of Candida albicans with the use of biogenic gold nanoparticles. Photochem Photobiol. 93:1081-1090. doi:10.1111/php.2017. 93.issue -4

Miller MW. 2000. Gene transfection and drug delivery. Ultrasound Med Biol. 26:S59-62. doi:10.1016/S0301-5629 (00)00166-6

Mima EG, Pavarina AC, Dovigo LN, Vergani CE, Costa CAS, Kurashi C, Bagnato VS. 2012. Susceptibility of Candida albicans to photodynamic therapy in a murine modelo of oral candidosis. Oral Surg Oral Med Oral Pathol Oral Radiol Endod. 109:392-401.

Nakonechny F, Nisnevitch M, Nitzan Y, Nisnevitch M. 2013. Sonodynamic excitation of Rose Bengal for eradication of gram-positive and gram-negative bacteria. Biomed Res Int. 2013:684930

Paardekopper M, van de Broek PJ, de Bruijne AW, Elferink JG, Dubbelman TM, Van Steveninck J. 1992. Photodynamic treatment of yeast cells with the dye toluidine blue: allor-none loss of plasma membrane barrier properties. Biochim Biophys Acta. 1108:86-90. doi:10.1016/00052736(92)90117-5

Park JH, Moon YH, Bang IS, Kim YC, Kim SA, Ahn SG, Yoon JH. 2010. Antimicrobial effect of photodynamic therapy using a highly pure chlorine e6. Laser Med Sci. 25:705-710. doi:10.1007/s10103-010-0781-1

Peeters E, Nelis HJ, Coenye T. 2008. Comparison of multiple methods for quantification of microbial biofilms grown in microtiter plates. J Microbiol Methods. 72:157-165. doi:10.1016/j.mimet.2007.11.010

Pereira CA, Romeiro RL, Costa AC, Machado AK, Junqueira JC, Jorge AO. 2011. Susceptibility of Candida albicans,
Staphylococcus aureus, and Streptococcus mutans biofilms to photodynamic inactivation: an in vitro study. Lasers Med Sci. 26:341-348. doi:10.1007/s10103-010-0852-3

Peres-Bota D, Rodriguez-Villalobos H, Dimopoulos G, Melot C, Vincent JL. 2004. Potential risk factors for infection with Candida spp. in critically ill patients. Clin Microbiol Infect. 10:550-555. doi:10.1111/j.14690691.2004.00873.x

Pfaller MA, Diekema DJ. 2007. Epidemiology of invasive candidiasis: a persistent public health problem. J Clin Microbiol Rev. 20:133-163. doi:10.1128/CMR.00029-06

Quishida CC, Carmello JC, Mima EG, Bagnato VS, Machado AL, Pavarina AC. 2015. Susceptibility of multispecies biofilm to photodynamic therapy using Photodithazine. Lasers Med Sci. 30:685-694. doi:10.1007/s10103-013-1397-z

Ramage G, Mowat E, Jones B, Williams C, Lopez-Ribot J. 2009. Our current understanding of fungal biofilms. Crit Rev Microbiol. 35:340-355. doi:10.3109/104084109032 41436

Rosenthal I, Sostaric JZ, Riesz P. 2004. Sonodynamic therapya review of the synergistic effects of drugs and ultrasound. Ultrason Sonochem. 11:349-363.

Samaranayake LP, MacFarlane TW. 1981. A retrospective study of patients with recurrent chronic atrophic candidosis. Oral Surg Oral Med Oral Pathol. 52:150-153. doi:10.1016/00304220(81)90312-1

Serpe L, Giuntini F. 2015. Sonodynamic antimicrobial chemotherapy: first steps towards a sound approach for microbe inactivation. J Photochem Photobiol B. 150:44-49. doi:10.1016/j.jphotobiol.2015.05.012

Spagnul C, Turner LC, Boyle RW. 2015. Immobilized photosensitisers for antimicrobial applications. J Photochem Photobiol B. 150:11-30. doi:10.1016/j.jphotobiol.2015. 04.021

Stepanovic S, Vukovic D, Dakic I, Savic B, Svabic-Vlahovic M. 2000. A modified microtiter-plate test for quantification of staphylococcal biofilm formation. J Microbiol Methods. 40:175-179. doi:10.1016/S0167-7012(00)00122-6

Strakhovskaia MG, Belenikina NS, Ivanova EV, Chemeris IK, Stranadko EF. 2002. The photodynamic inactivation of Candida guilliermondii in the presence of photodithazine. Mikrobiologiia. 71:349-353.

Sudbery PE. 2011. Growth of Candida albicans hyphae. Nat Rev Microbiol. 9:737-748. doi:10.1038/nrmicro2636

Thompson DS, Carlisle PL, Kadosh D. 2011. Coevolution of morphology and virulence in Candida species. Eukaryot Cell. 10:1173-1182. doi:10.1128/EC.05085-11

Umemura S, Yumita N, Umemura K, Nishigaki R. 1999. Sonodynamically induced effect of rose bengal on isolated sarcoma 180 cells. Cancer Chemother Pharmacol. 43:389393. doi:10.1007/s002800050912

Wang X, Ip M, Leung AW, Xu C. 2014. Sonodynamic inactivation of methicillin-resistant Staphylococcus aureus in planktonic condition by curcumin under ultrasound sonication. Ultrasonics. 54:2109-2114. doi:10.1016/j. ultras.2014.06.017

White TC, Marr KA, Bowden RA. 1998. Clinical, cellular, and molecular factors that contribute to antifungal drug resistance. Clin Microbiol Rev. 11:382-402.

Zhuang D, Hou C, Bi L, Han J, Hao Y, Cao W, Zhou Q. 2014. Sonodynamic effects of hematoporphyrin monomethyl ether on Staphylococcus aureus in vitro. FEMS Microbiol Lett. 361:174-180. doi:10.1111/fml.2014.361.issue-2 